\title{
Automated ECG Diagnosis
}

\author{
Upasani D.E. ${ }^{1}$, Dr.R.D.Kharadkar ${ }^{2}$ \\ ${ }^{1}$ (Researcher Bharati Vidyapeeth, Pune $)^{2}$ (Principal, GHRIET, Pune)
}

\begin{abstract}
Myocardial ischemia \& other cardiac disorder diagnosis using long duration electrocardiographic recordings is a simple and non-invasive method that needs further development in order be used in the everyday medical practice. Several techniques that automate ischemia\& other cardiac detection have been proposed during the last decade which are under evaluation. They are based on different methodological approaches, which include digital signal analysis, rule-based techniques, fuzzy logic methods and artificial neural networks, with each one of them exhibiting its own advantages and disadvantages. Most recent systems employ hybrid system (combination of ANN \& Fuzzy logy) to perform diagnosis since they have demonstrated great consistency in producing accurate results. The performance of the developed detection systems is very promising but they need further evaluation
\end{abstract}

Keywords:- ANN, arrhythmia, ECG, Ischemia, tachycardia,

\section{INTRODUCTION}

Clinically, biomedical signals are primarily acquired for monitoring (detecting or estimating) specific pathological/physiological states for purposes of diagnosis and evaluating therapy. In some cases of basic research, they are also used for decoding and eventual modelling of specific biological systems. Furthermore, current technology allows the acquisition of multiple channels of these signals. This brings up additional signal-processing challenges to quantify physiologically meaningful interactions among these channels. Goals of signal processing in all these cases usually are noise removal, accurate quantification of signal model and its components through analysis (system identification for modelling and control purposes), feature extraction for deciding function and prediction of future pathological or functional events as in substitute devices for heart. Typical biological applications may involve the use of signal-processing algorithms for more than one of these reasons. The monitored biological signal in most cases is considered an additive combination of signal and noise. Noise can be from instrumentation (sensors, amplifiers, filters, etc.), from electromagnetic interference (EMI), or in general, any signal that is asynchronous and uncorrelated with the noise characteristics, which will eventually lead to an appropriate choice of signal-processing method. The main objective of automated ECG analysers is to assist in making a diagnosis. Obviously, they will not replace medical experts, but their decision can be considered as an unbiased second opinion. The automated diagnosis systems developed so far are not reliable in detection of ischemic episodes, fibrillations, and recognition of arrhythmias and are having number of limitations and short comings. In recent years, computer assisted ECG interpretation has played an important role in automatic diagnosis of heart abnormalities [1, 3]. The ECG wave forms ; reflects the physical condition of human heart and is the most important biological signal to study and diagnose cardiac abnormalities, disturbances. So, it is important to record the patient's ECG for a long period of time for clinical diagnosis. The clinical significance diagnosis depends on different parameters of ECG; complex QRS, wave P, frequency, Heart Rate Variability R-R, etc. In these applications, it is more important to develop signal processing methods that permit real time feature extraction and de-noising of the ECG characteristic. The extracted parameters are used for the classification of the cardiac pathologies and make an automatic tool of diagnosis in the services of doctors before the arrival of a quantified patient.

Table 1. ECG Characteristics:

\begin{tabular}{|c|c|c|c|c|}
\hline $\begin{array}{c}\text { Assoc } \\
\text { iated } \\
\text { Wave }\end{array}$ & $\begin{array}{c}\text { Duration } \\
\text { (Sec) }\end{array}$ & $\begin{array}{c}\text { Amplitu } \\
\text { de (mV) }\end{array}$ & Frequency & $\begin{array}{c}\text { Mechani } \\
\text { cal } \\
\text { Action }\end{array}$ \\
\hline $\begin{array}{c}\mathrm{P} \\
\text { wave }\end{array}$ & $<0.12$ & $\leq 0.3$ & 10 & $\begin{array}{c}\text { Atrial } \\
\text { Depolari } \\
\text { zation }\end{array}$ \\
\hline $\begin{array}{c}\text { QRS } \\
\text { Comp } \\
\text { lex }\end{array}$ & $\begin{array}{c}0.08 \text { to } \\
0.12\end{array}$ & 0.5 to 2 & 20 to 50 & $\begin{array}{c}\text { Depolari } \\
\text { zation of } \\
\text { Ventricle } \\
\text { s }\end{array}$ \\
\hline $\begin{array}{c}\mathrm{T} \\
\text { wave }\end{array}$ & 0.2 & 0.2 & 5 & $\begin{array}{c}\text { Repolari } \\
\text { zation of } \\
\text { Ventricle } \\
\text { s }\end{array}$ \\
\hline
\end{tabular}

\section{REVIEW OF LITERATURE:}

Sokolow et al., (1990), indicate that the state of cardiac health is generally reflected in the shape of the ECG waveform and heart rate. Cuiwei Li et al., (1995) showed that it is easy with multi scale information / decomposition in wavelets transformation to characterize the ECG waves. Khadra et al., (1997) proposed a classification of life threatening cardiac arrhythmias using wavelet transforms. MG Tsipouras et al., (2004) used time frequency analysis for classification of atrial tachya arrhythmias. Al-Fahoum and Howit (1999) joint radial basis neural networks to wavelet transformation to classify cardiac arrhythmias. 
Weissan et al., (1990); Akselord et al., (1981); Pomeranz et al., (1985) showed that the spectral analysis is the essential linear techniques used for the HRV signals analysis. Silipo et al., (1998) has shown that the ANN's approach is shown to be capable of dealing with the ambiguous nature of the ECG signal when tested and compared with the most common traditional ECG analysis on appropriate data bases [4]. Ali Shahidi Zandi et al., (2005) used a method based on the continuous Wavelet transform and Artificial Neural Network for detection of ventricular late potentials in HighResolution ECG signals. Mei Jiang Kong et al., (2005) used block-based neural networks to classify ECG Signals. Fira et al., (2008) proposed an ECG compressed technique and its validation using NN's. The choice of the wavelet family as well as the selection of the analyzing function and level decomposition into these families have been discussed to the Daubechies decompositions provided by the Daubechies wavelet (level 3), the coiflet wavelet (level 6) and the symmetric wavelet (level 6) [5]. Another important threatening abnormality that causes sudden death is cardiac arrhythmia. Arrhythmic patterns are characterized by a variety of morphologies, which complicate their automated classification. The classification process in most systems is based on the evaluation of several ECG features. Arrhythmias and specifically premature ventricular complexes can be identified with the use of matched filters [6]. Parametric modelling was also used in arrhythmia detection, and specifically an auto-regressive model was applied for the recognition of ventricular tachycardia (slow heart rate) patients [7]. Atrial fibrillation, on the other hand, can be detected using the Wigner-Ville transform, which takes advantage of the fact that the time variations of this type of arrhythmia are limited in specific frequency bands [8]. Another time-frequency analysis approach employs a distribution with a logarithmic frequency scale for each spectrum in order to estimate a representative spectral profile and classify atrial arrhythmias [9].

\section{METHODS USED}

1. Digital signal processing,

2. Knowledge based system,

3. A Rule-based system

4. Fuzzy Expert system,

5. Artificial Neural Networks

6. Hybrid system

DSP Based Signal Processing: It transforms the acquired ECG signal in order to reveal any discriminative information. Using the Fourier transform, the signal is analyzed in the time-frequency domain. The wavelet transform, on the other hand, analyzes the signal in the timescale domain, using different scales of the same wavelet. In general, the wavelet transform can be considered as a filter bank of band-pass filters. Nonlinear ECG analysis produces new indexes capable of detecting the pathological heart conditions. Principal component analysis (PCA), or Karhunen-Loe've transform, is a nonlinear method used for feature extraction and diagnosis as well. Ischemia detection using digital signal analysis techniques is based on the transformation of the original recorded signal and the extraction of new indexes capable of diagnosing myocardial ischemia. These techniques analyze the ECG signal in the time or/and the frequency domain, and, based on this analysis, they extract useful features that are used to perform diagnosis.

Knowledge Based System: It employs medical knowledge in their decision-making process. This medical knowledge is expressed by a set of rules that is applied on the ECG features. Usually, a representative template is employed that is constructed after averaging a number of cardiac beats. All the beats are compared with this template and classified accordingly (template matching). Another approach is to use the template for feature extraction. The feature values are introduced to the set of rules and the produced classification refers to the group of the beats that constitute the template. The rules employed in the knowledge-based systems correspond to inflexible thresholds. Near the threshold value, the classification is realized without taking into account the noise distortion or the subjectivity of each patient. Other knowledge-based systems use rules that express the probability of ischemia using information extracted from the patient's history and the ECG recordings [10]. The use of data from the patient's history may increase the efficiency of a diagnosis system but requires proper selection and analysis.

Rule Based System: Rule-based systems employ sets of rules that are derived from medical knowledge in order to evaluate the extracted ECG features and detect the ischemic episodes Rule based methods can also be used to classify arrhythmias as:

(1) Normal sinus rhythm;

(2) Premature atrial, nodal, and supra ventricular contractions (rapid heart-beat originating in atria);

(3) Premature ventricular contractions;

(4) Escape; and

(5) Ventricular flutter/fibrillation.

A variety of ECG features can also be used, extracted from the $\mathrm{P}$ wave, the $\mathrm{R}$ wave, the QRS complex, the PR interval, the $\mathrm{R}-\mathrm{R}$ interval, and the $\mathrm{P}-\mathrm{P}$ interval. Based on a decision tree, all types of arrhythmias can be identified [11]. Although this approach seems very promising, its performance is affected by the presence of noise, thus proper filtering should be employed. The rules employed in the rule - based systems correspond to inflexible thresholds; this being the main disadvantage of these systems. Near the threshold value the classification is realized without taking into account the noise distortion or the subjectivity of each patient.

Fuzzy Expert System : These are more or less an extension of the rule-based systems, designed to deal with diagnosis problem. They employ similar sets of rules, but their logic is not two-valued (yes or no) but fuzzy, which allows the representation of possibility (called fuzziness) through membership functions concerning the decision suggested by a rule, which simulates better human-like reasoning and, consequently, the diagnostic chain of thoughts followed by physicians. 
Artificial Neural Network: Artificial Neural Network is biologically inspired network that are suitable for classification of biomedical data. Especially for the ECG signal, ANNs can be used for Data analysis, Knowledge discovery, Rule extraction, Prediction and other tasks. ANN models combined with wavelet theory have been tested on ventricular tachycardia recognition [12]. In general, the wavelet network can be considered as a multilayer perceptron with the wavelet nodes acting as processing units for feature extraction. During the training process, the network parameters (weights and biases), as well as the wavelet parameters (scale, shift, and frequency), are modified. In the wavelet nodes, the Morlet wavelets can be used to process the information from the QRS complex. The architecture of the network should not be complex in order to avoid training difficulties. The limitations of ANN are some of them suffer from long processing times for training, and their structural complexity grows as the size of the input vector increases. The other common problem is that they only analyse one part of the signal and hence can detect only one or two abnormalities [13]

\section{PROPOSED SYSTEM:}

This method describes an Intelligent Diagnosis System using Hybrid approach of Adaptive Neuro-Fuzzy Inference System (ANFIS) model for classification of Electrocardiogram (ECG) signals, and comparison this Technique with Feed-Forward Neural Network (FFNN), and Fuzzy Inference Systems (FIS). Feature extraction using Independent Component Analysis (ICA) and power spectrum, together with the RR interval then serve as input feature vector, this feature were used as input of FFNN, FIS, and ANFIS classifiers. Six types of ECG signals: Normal Sinus Rhythm (NSR), Premature Ventricular Contraction (PVC), Atrial Premature Contraction (APC), Ventricular Tachycardia (VT), Ventricular Fibrillation (VF) and Supraventricular Tachycardia (SVT) can be detected using hybrid approach. Fuzzy Hybrid Neural Network composed of two sub-networks connected the Fuzzy Self- Organizing layer performing the pre-classification task and the following Multilayer Perceptron (MLP) working as the final classifier. In [14] used largest Lyapunov exponent, spectral entropy and Poincare geometry for ECG feature extraction, Adaptive Neuro-Fuzzy Inference System (ANFIS) was presented for classification of the ECG signals. In [8] used Wavelet Transform for ECG feature extraction, There are two classifiers that used Analytical classifier and ANFIS classifier. In [9] used Lyapunov exponents for ECG feature extraction, Adaptive Neuro-Fuzzy Inference System (ANFIS) were presented for classification of the ECG signals. In [15] used three different techniques to extract features from ECG signal which are Fast Fourier Transform, Autoregressive Modelling, and Principal Component Analysis. The features were be classified by using three different classifiers which are ANN, and two types of statistical classifiers which are Minimum Distance Classifier, Bayes Minimum Distance Classifier. Another similar approach uses the wavelet transform and specifically a quadratic spline wavelet to extract features and a fuzzy ANN to identify ventricular premature contractions
[16].Arrhythmia classification has also been addressed with an auto-associative ANN [17]. This network is trained using the beats contained in the initial $15 \%$ of the ECG. Specifically, the $150 \mathrm{~ms}$ ECG interval that starts from the beginning of the QRS complex and information extracted from the R-R interval are used as input. Furthermore, using the whole cardiac beat as input, premature ventricular QRS complexes can be identified with a feed forward ANN [18]. "Mixture of experts" is an ANN approach for arrhythmia recognition, which uses an already trained ANN [19-23]. When new cardiac patterns are introduced to the system, an updating process is realized using the self-organizing map and the learning vector quantization algorithms. Automated ECG diagnosis system consists of a series of procedures that can be used in order for useful clinical information to be produced. This will help the physician to reach a diagnosis faster and safer, concerning the pathophysiological condition of the patient's heart.

The system can be divided in four stages:

(1) Signal acquisition and pre-processing,

(2) Signal monitoring and processing,

(3) Feature extraction,

(4) Diagnosis.

Signal acquisition and pre-processing should full fill certain specifications concerning the sampling frequency $(100 \mathrm{~Hz}-1$ $\mathrm{KHz}$ ), the resolution (number of bits for each sample), and the sensitivity, which expresses the signal's amplitude range (usually $5 \mathrm{mV}$ or $6 \mathrm{mV}$ ). In its digital form, the ECG signal is processed, monitored and filtering is performed to suppress noise and enhance the relevant ECG characteristics. The ECG feature extraction follows the processing stage. In this stage, all the relevant ECG characteristics are recognized and some of their features are estimated. The extracted features vary from simple ones, like the duration and amplitude of some ECG characteristics, to more complex ones, like slopes, intervals, frequencies, or other discriminating indices, which are used in the diagnosis stage because the values of certain features are indicators of the existence of an underlying disease. Apparently, the measurement accuracy is important at this stage, and computerized methods can be used to address it efficiently. The last stage is the automated ECG diagnosis, where explicit medical knowledge will be used. The collaboration with medical experts is necessary, as the individual characteristics of each patient complicate the decision-making task. Various automated approaches can be used for this stage having advantages and disadvantages. The system will be able to detect the abnormalities in the ECG and may also produce interpretations for the decisions made. 


\section{RESULTS:}

ECG Signal Generated for different Conditions in LAB VIEW

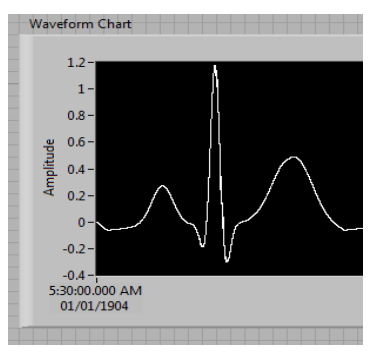

Fig1: Normal ECG
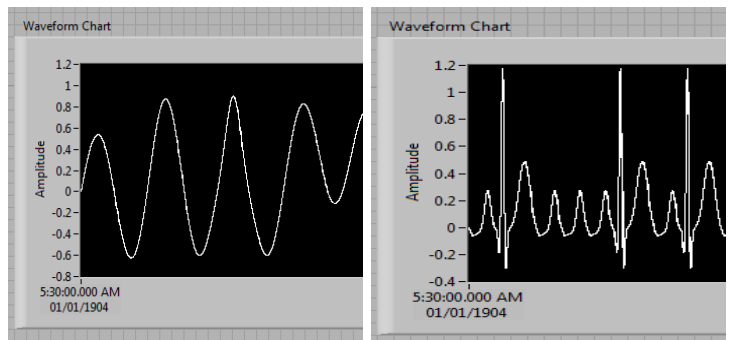

Fig.3:Atrial Fibrillation Fig.4:Ventricular Fibrillation

\begin{tabular}{|l|l|l|l|}
\hline $\begin{array}{l}\text { Ischemia } \\
\text { Detection } \\
\text { Systems }\end{array}$ & $\begin{array}{l}\text { Positive } \\
\text { Prediction } \\
\text { Accuracy }\end{array}$ & $\begin{array}{l}\text { Arrhythmia } \\
\text { Classification } \\
\text { Systems }\end{array}$ & PPA \\
\hline $\begin{array}{l}\text { Digital Signal } \\
\text { Processing }\end{array}$ & $86 \%$ & $\begin{array}{l}\text { Digital Signal } \\
\text { Processing }\end{array}$ & $89 \%$ \\
\hline $\begin{array}{l}\text { Knowledge- } \\
\text { Based }\end{array}$ & $79 \%$ & $\begin{array}{l}\text { Knowledge- } \\
\text { Based }\end{array}$ & $93 \%$ \\
\hline Fuzzy Logic & $68 \%$ & Fuzzy Logic & $81 \%$ \\
\hline $\begin{array}{l}\text { Artificial } \\
\text { Neural } \\
\text { Network }\end{array}$ & $89 \%$ & $\begin{array}{l}\text { Artificial } \\
\text { Neural } \\
\text { Network }\end{array}$ & $78 \%$ \\
\hline $\begin{array}{l}\text { Hybrid } \\
\text { System }\end{array}$ & $90 \%$ & Hybrid System & $95 \%$ \\
\hline
\end{tabular}

\section{CONCLUSION :}

Technique used in previous studies found that the use of hybrid techniques is the most successful technique from others, so the Hybrid Technique can be used for automated diagnosis of ECG. Techniques like Independent Component Analysis (ICA) and power spectrum, etc. has to be used along with Hybrid techniques to extract the Important Feature from the ECG signal.

\section{REFERENCES:}

[1]. M.G. Tsipouras, V.P. Oikonomou, D.I. Fotiadis,L.K. Michlis, D. Sideris.2004.Classification of Atrial Tachyrrhythmias in Electrocardiograms Using Time Frequency Analysis. Computers in Cardiology. 31: 245-248. 2004 IEEE.

[2]. B. Anuradha, V. Reddy. 2008. Cardiac Arhythmia Classification Using Fuzzy Classifiers. Journal of Theoretical and Applied Information Technology.
[3]. M.G. Tsipouras, C. Voglis, I. E Lagaris and D. I.Fotiadisohet. 2005. Cardiac Arrhythmia Classification Using Support Vector Machines.

[4]. Senhadi L, Carrault G, Bellanger J. J. and Passariello G. 1995. Comparing Wavelet Transforms for Recognizing Cardiac Patterns. IEEE Engineering in Medicine and Biology Magazine. 14(2): 167-173.

[5]. A. Kachouri, M. Ben Messaoud and A. Dallali. 2009. Wavelet based on Electrocardiogram Signal Analysis for Classification and Diagnosis By Neural Networks. SSD'03. March 26-28. Sousse, Tunisia.

[6]. G. B. Moody and R. G. Mark, Development and evaluation of a 2-lead ECG analysis program. Proc.IEEE Comput. Cardiol. 1982: 39-44.

[7]. [H. F. Schels, R. Haberl, G. Jilge, P. Steinbigler, and G. Steinbeck, Frequency analysis of the electrocardiogram with maximum entropy method for identification of patients with sustained ventricular tachycardia. IEEE Trans. Biomed. Eng. 1991; BME- 38:821- 826.

[8]. M. Stridh, L. Sornmo, C. J. Meurling, and S. B. Olsson, Sequential characterization of atrial tachyarrhythmia's based on ECG time-frequency analysis. IEEE Trans. Biomed. Eng. 2004; 51:100114.

[9]. [Fokapu O. and Girard J.P. 1994. Evolution temporelle et fréquentielle de l'ECG: analyse B attement par battement, ITBM. 14(1): 102-112.

[10]. C. B. Cairns, J. T. Niemann, and H. P. Selker,Computerised version of the timeinsensitivepredictive instrument: use of the $\mathrm{Q}$ wave, ST-segment, $\mathrm{T}$ wave, and patient history in the diagnosis of acute myocardial infarction by the computerized ECG. J. Electrocardiol. 1992; 24:S46S49.

[11]. Margarita Sordo. 2002. Introduction to Neural Networks in Healthcare-A review.

[12]. Chouakri S. A., Bereksi-Reguig F., Ahmaïdi S. and Fokapu O. 2005. Wavelet Denoising of the Electrocardiogram Signal Based on the Corrupted Noise Estimation. Computers in Cardiology. 32: 1021-1024.

[13]. Wei Jiang Kong S. G., Peterson G. D. 2005. ECG signal classification using block-based neural networks. Proc. IJCNN. p. 326

[14]. M. Kundu, M. Nasipuri, and D. K. Basu, A knowledge-based approach to ECG interpretation using fuzzy logic. IEEE 16 ELECTROCARDIOGRAM (ECG): (AUTOMATED DIAGNOSIS) Trans. Syst. Man Cybernet. Part B: Cybernet. 1998; 28:237- 243.

[15]. R. Silipo, R. Vergassola, W. Zong, and M. R. Berthold, Knowledge-based and data-driven models in arrhythmia fuzzy classification. Meth. Inf. Med. 2001; 40:397-402.

[16]. O. Wieben, V. X. Afonso, and W. J. Tompkins, Classification of premature ventricular complexes using filter bank features, induction of decision trees and a fuzzy rule-based system. Med. Biol. Eng.Comput. 1999; 37:560-565. 
[17]. H. Dickhaus and H. Heinrich, Classifying biosignals With wavelet networks-a method for noninvasive diagnosis. IEEE Eng. Med. Biol. 1996; 15:103-111

[18]. N. Kannathal, C.M. Lima, U. Rajendra Acharya and P.K. Sadasivan, "Cardiac state diagnosis using adaptive neuro-fuzzy technique", Medical Engineering \& Physics, Singapore, 2006.

[19]. Elif Derya Ubeyli, "Adaptive neuro-fuzzy inference system for classification of ECG signals using Lyapunov exponents",computer methods and programs in biomedicine, Ankara, Turkey,

[20]. M. Mansor ,"Electrocardiogram (ECG) Classification Using Artificial Neural Networks," Master thesis, Department of Biomedical Engineering, Faculty of Engineering, Helwan Universty, Cairo, Egypt, 2007.

[21]. L. Y. Shyu, Y. H. Wu, and W. Hu, Using wavelet transform and fuzzy neural network for VPC detection from the Holter ECG. IEEE Trans. Biomed. Eng. 2004; 51:1269-1273.

[22]. R. Silipo and C. Marchesi, Artificial neural networks for automatic ECG analysis. IEEE Trans.Sign. Proc. 1998; 46:1417-1425.

[23]. N. Maglaveras, T. Stamkopoulos, C. Pappas, and M. Strintzis, ECG processing techniques based on neural networks and bidirectional associative emories. $J$. Med. Eng. Technol. 1998; 22:106-111. 